\title{
The Efficacy of Bupivacaine vs Bupivacaine with Dexamethasone for Supraclavicular Brachial Plexus Block in Patients Undergoing Upper- Limb Surgeries
}

\author{
Khalid Ali Khaleel MBChB, DA FICMS ${ }^{1}$, Zaid Al-Attar MBChB, $\mathrm{PhD}^{2 *}$
}

${ }^{1}$ Senior anesthesiologist in Dept. of Anesthesia, Intensive Care and Pain Management, Neuroscience hospital / Baghdad /Irac

${ }^{2}$ Lecturer, Dept. of Pharmacology, Al-Kindy College of Medicine University of Baghdad Iraq

Objective: Brachial plexus block using bupivacaine as compared to general anesthesia is regarded as a valuable alternative as it induces adequate muscle relaxation, excellent analgesia intra and post operatively. Dexamethasone was combined with bupivacaine to study its effects in terms of onset and duration of sensory and motor block after their administration in patients undergoing upper limb surgeries by implementing the supraclavicular block. Materials and methods: Brachial plexus block is a viable alternative to general anesthesia as it provides adequate muscle relaxation, excellent intraoperative and post-operative analgesia. Dexamethasone, a synthetic glucocorticoid was combined with local anesthetics to study the effects. This study was conducted to compare the onset and duration of sensory and motor block following administration of either bupivacaine and bupivacaine- Dexamethasone in patients undergoing upper limb surgeries under USG guided supraclavicular block. Materials and methods: 100 ASA II, III, IV patients between of 18 and 79 years who underwent elective upper limb surgeries randomly allocated into two groups of 50 each by a sealed envelope technique to receive $20 \mathrm{ml}$ of $0.5 \%$ Bupivacaine and $2 \mathrm{ml}$ of normal saline in Group BS and $20 \mathrm{ml}$ of $0.5 \%$ Bupivacaine with $2 \mathrm{ml}(8 \mathrm{mg})$ of Dexamethasone in Group BD. The onset and duration of sensory and motor blockade were observed between the two groups in addition to analgesic effect duration. Results: The onset of sensory and motor blockade was faster in Group BD when compared to Group BS (p<0.0001). The duration of sensory and motor blockade was longer in Group BD when compared to Group BS ( $<<0.0001$ ). There were no significant hemodynamic changes in the study group. Conclusion: Dexamethasone when added to Bupivacaine in supraclavicular block shortens the onset time and prolongs the duration of sensory and motor blockade without any systemic side effects.

Keywords: Brachial plexus block, dexamethasone, supraclavicular, bupivacaine, upper- limb surgeries.

Copyright @ 2020: This is an open-access article distributed under the terms of the Creative Commons Attribution license which permits unrestricted use, distribution, and reproduction in any medium for non-commercial use (NonCommercial, or CC-BY-NC) provided the original author and source are credited.

\section{INTRODUCTION}

Surgeries involving the upper limb are usually done under general anesthesia which has its drawbacks as airway instrumentation, multiple drugs exposure, and aspiration chance. On the other hand, brachial plexus block (BPB) is now regarded as a promising alternative that is devoid of the above-mentioned complications [1]. Administering BPB blindly has the risk of failure and complications. Yet, the introduction of ultrasound guided approach (USG) has raised interest in BPB.

In this study, we are using the supraclavicular block to provide anesthesia from fingers up to midarm. This approach has a rapid onset and high rate of success. The first supraclavicular block was performed by Kullenkampff in 1911-1912 [2]. Ultrasound- guided supraclavicular brachial plexus block is a safe, reliable anesthetic technique for upper- limb surgeries with less complications [1]. Ultrasound guidance leads to decreased procedure time, faster onset of action, and higher block success rates without neural injuries. Bupivacaine is a local anesthetic used in BPB that is lasting 3 to $6 \mathrm{hr}$ [1]. Such duration is enough to provide intraoperative but may be not enough for post-operative analgesia. Post-op analgesia requires rescue analgesia in the form of NSAIDS or opioids. Therefore, prolonging effect of bupivacaine would lessen the need for rescue analgesia and its complications or side effects. Several agents have been introduced to prolong the duration of action including epinephrine, magnesium sulphate, alpha 2 agonists, clonidine, and midazolam. Recently, 
corticosteroids have been studied. They induce some degree of vasoconstriction and thus they may reduce local absorption. Few studies have been investigated the role of dexamethasone on supraclavicular block showing that it prolongs the effect of local anesthetic while the duration of block is variable.

\section{AIM}

This study aims to investigate the effect of dexamethasone on supraclavicular block to address this knowledge gap.

\section{MATERIALS AND METHODS}

The present study was conducted in the Department of Anesthesiology, Medical City Hospital, Baghdad, Iraq between January 2018 and November 2019. The inclusion criteria for our study were American Society of Anesthesiologists (ASA) Grades II, III, and IV patients between the age group of 18 and 79 years and those undergoing elective upper- limb surgeries (i.e., elbow, forearm, and hand). Patients with bleeding disorders, respiratory compromise, known allergies to local anesthetics, and those with infections at the site of block or documented neuromuscular disorders were excluded from the study.

\section{Sample size (n)}

The total sample size was taken as 100. After obtaining institutional review board and ethical committee clearance, fifty patients were allocated in a randomized manner into one of the two groups.

Group BS: Will receive $20 \mathrm{ml}$ of $0.5 \%$ bupivacaine +2 $\mathrm{ml}$ of normal saline.

Group BD: Will receive $20 \mathrm{ml}$ of $0.5 \%$ bupivacaine +2 $\mathrm{ml}(8 \mathrm{mg})$ of dexamethasone.

Once the patient fulfilled the inclusion criteria, History, Clinical examination and Informed consent was taken from the patient. Every Patient was subjected to routine investigations like complete blood picture, RBS, Chest Xray and ECG. The anesthesiologist involved in the data collection as well as the patient was blinded to the content of the study solution.

Preoperatively, the patient's IV line was secured with either $18 \mathrm{G}$ or $20 \mathrm{G}$ cannula and IV ringer lactate solution was started at $5 \mathrm{ml} / \mathrm{kg} / \mathrm{h}$. The patient was then shifted to the operation theater and monitors such as ECG, $\mathrm{SpO}$, and blood pressure (BP) were recorded. The patient was placed in $30^{\circ}$ propped up position with face turned to opposite side and ipsilateral upper arm placed by the side of the patient. Under strict aseptic precautions, the area between the mandible and the ipsilateral nipple was painted and draped. The carotid artery was palpated and a skin wheal was raised by injecting $2 \mathrm{ml}$ of lidocaine $2 \%$.
Under ultrasound guidance, an echogenic needle was used to localize the brachial plexus which is present posterolateral to the brachial plexus. An echogenic needle used was inserted from the right side of the probe for the right-side shoulder and vice versa. Aspiration was done for checking the absence of blood. The echogenic needle was inserted into the nerve sheath under ultrasonologic view. The injected volume gently expands the connective tissue surrounding the nerves which is called hydro dissection. This allows the needle a clear path. Sensory block was assessed by pinprick test using a 3 - point scale as follows:

- $0=$ sharp pin felt

- $1=$ dull sensation felt (analgesia)

- 2 = no sensation felt (anesthesia).

Motor block was assessed by thumb abduction (radial nerve), thumb adduction (ulnar nerve), thumb opposition (median nerve), and flexion at the elbow (musculocutaneous nerve) on a 3 - point scale for motor function which are described as follows:

- $0=$ normal motor function with full flexion and extension of elbow, wrist, and fingers

- 1 = reduced motor strength but able to move fingers

- 2 = complete motor blocks with an inability to move fingers. Sensory and motor block was assessed every 3 min until $30 \mathrm{~min}$ after injection, and then every $30 \mathrm{~min}$ after the surgery, until they had resolved. Onset time was defined as the time interval between the end of total local anesthetic administration and complete sensory block. Complete sensory block was defined by anesthetic block (score 2) on all nerve territories. Duration of sensory block was defined as the time interval between the end of local anesthetic administration and the complete resolution of effect of anesthesia on all nerves.

Onset of motor block was defined as the time interval between administrations of local anesthetic solution to loss of movements. Complete motor block was defined as the absence of voluntary movements in hand and forearm (score 0). Duration of motor block was defined as the time interval between the end of local anesthetic administration and the recovery of complete motor function of the hand and forearm. Heart rate, systolic arterial $\mathrm{BP}$, and diastolic arterial $\mathrm{BP}$ were recorded at $0,5,10,15,30,45,60,90$, and $120 \mathrm{~min}$. adverse events such as hypotension were defined as decrease in systolic BP by $20 \%$ from baseline values.

Pain was assessed using a visual analog scale (VAS) (0-10). Nursing staff administered intramuscular diclofenac $75 \mathrm{mg}$ when VAS $>4$.

\section{STATISTICAL ANALYSIS}

All the data were expressed as mean \pm standard deviation. Mann-Whitney test was implemented to for statistical comparisons using $\mathrm{P}<$ 0.05 as statistically significant. 


\section{RESULTS}

A hundred patients were randomly allocated into two groups of fifty each:

- Group BS: Received $20 \mathrm{ml}$ of $0.5 \%$ bupivacaine +2

$\mathrm{ml}$ of normal saline

- Group BD: Received $20 \mathrm{ml}$ of $0.5 \%$ bupivacaine +2 $\mathrm{ml}$ (8 mg) dexamethasone.
Figure 1: shows a comparison between the two groups in terms of mean and standard deviation. They are very similar. Moreover, there is no statistically significant difference $p>0.05$. Which means that the groups are very similar and thus can be used for statistical comparisons

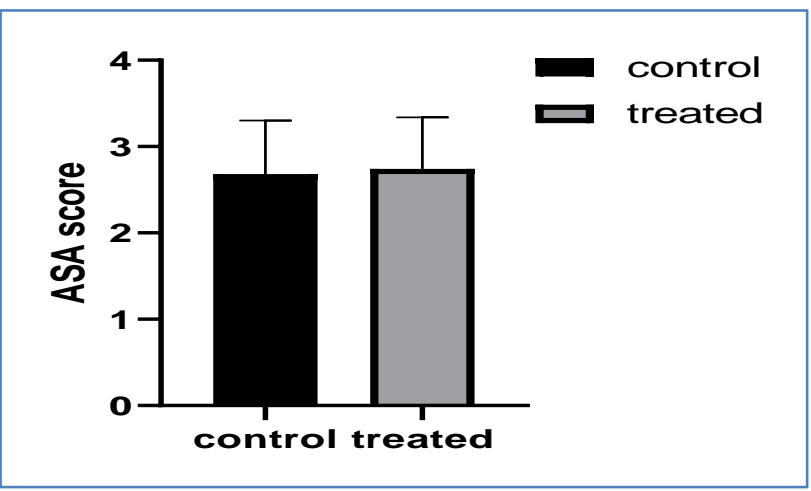

Fig-1: The ASA scores of the control and the treated groups. The Mean and the standard deviation of both groups are illustrated. They are nearly similar and there is no significant difference $p>0.05$ using Mann-Whitney test

Bupivacaine and dexamethasone has shown faster onset and longer duration of sensory block as compared with bupivacaine and normal saline (figure 2A, B) that is statistically significant $\mathrm{P}<0.001$.
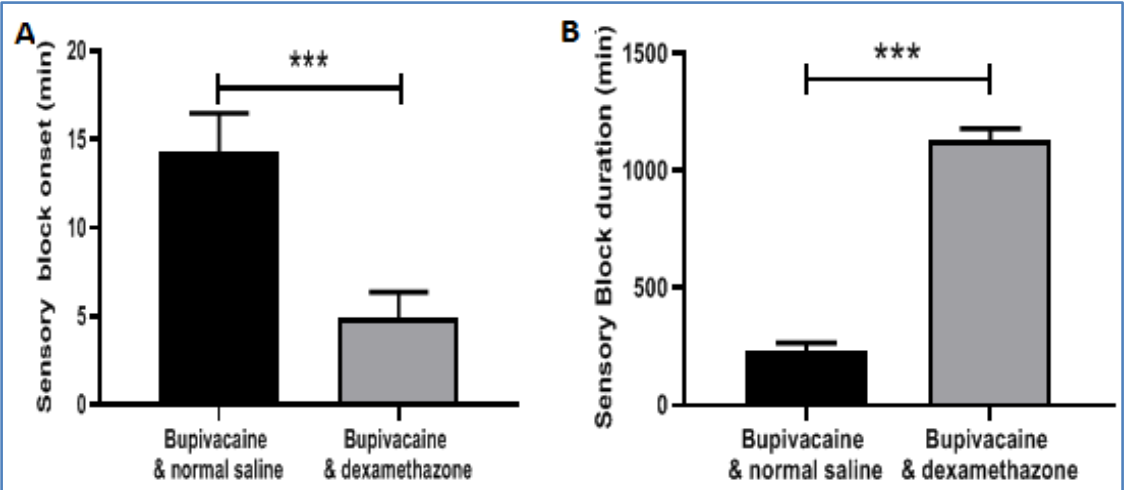

Fig-2 A: Bupivacaine with normal saline vs bupivacaine and dexamethasone in terms of sensory block onset. Bupivacaine and dexamethasone shows a statistically significant difference $\mathbf{P}<0.001 .2 \mathrm{~B}$ : Bupivacaine with normal saline vs bupivacaine and dexamethasone in terms of sensory block duration. Bupivacaine and dexamethasone shows a statistically significant difference $P<0.001$

Similarly, Bupivacaine and dexamethasone has shown faster onset and longer duration of motor block as compared with bupivacaine and normal saline (figure $3 \mathrm{~A}, \mathrm{~B})$ that is statistically significant $\mathrm{P}<0.001$.
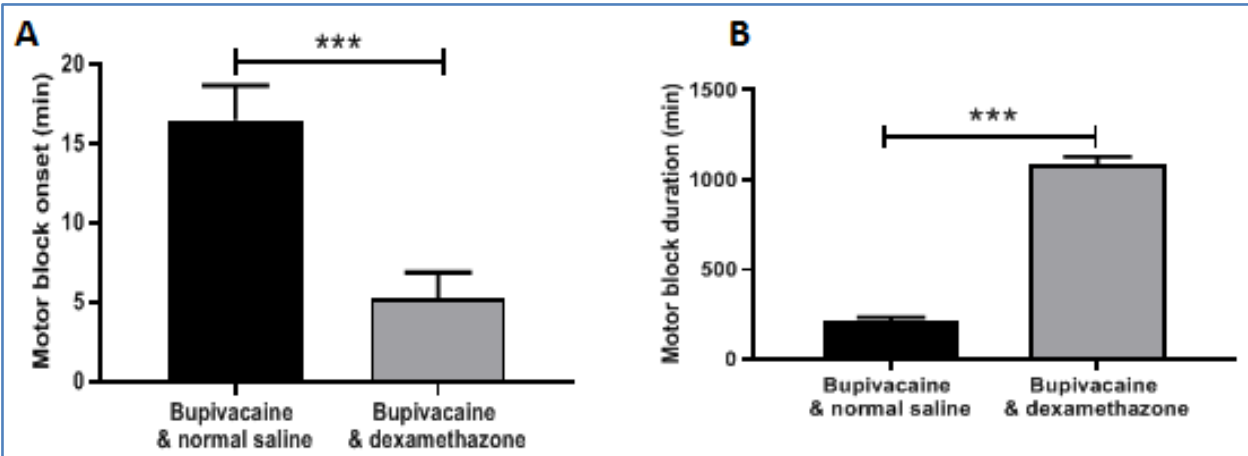

Fig-3 A: Bupivacaine with normal saline vs bupivacaine and dexamethasone in terms of motor block onset. Bupivacaine and dexamethasone shows a statistically significant difference $P<0.001$. 3 B: Bupivacaine with normal saline vs bupivacaine and dexamethasone in terms of motor block duration. Bupivacaine and dexamethasone shows a statistically significant difference $\mathbf{P}<0.001$ 
In terms of analgesic efficacy, bupivacaine and dexamethasone shows a statistically significant difference $\mathrm{P}<0.001$ as compared with bupivacaine with normal saline

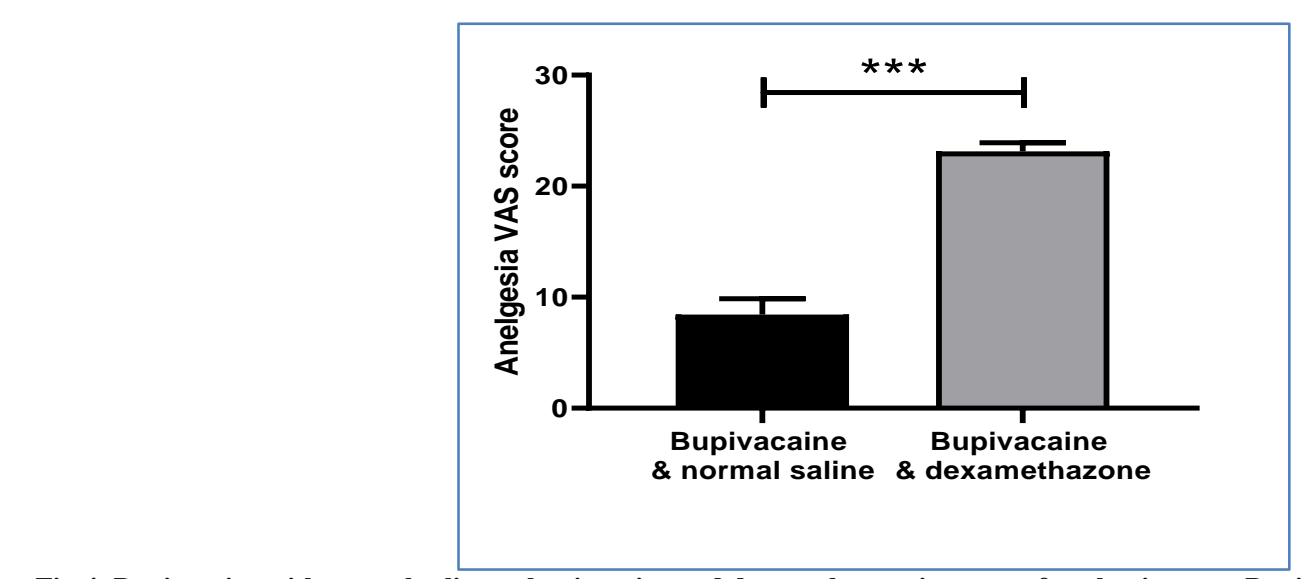

Fig-4: Bupivacaine with normal saline vs bupivacaine and dexamethasone in terms of analgesia score. Bupivacaine and dexamethasone shows a statistically significant difference $P<0.001$.

\section{DISCUSSION}

Supraclavicular block is a commonly performed anesthetic technique for patients undergoing surgeries on the upper limb. It blocks the plexus at the level of trunks leading to high rates of success, thus making it a popular choice among anesthesiologists. The addition of USG has revolutionized the field of regional anesthesia. It has increased the success rates of peripheral nerve blocks and avoids complications such as inadvertent intravascular injection, pneumothorax, and trauma to nerves, which were seen with the classical "Blind"/ "Landmark" techniques. It also helps to reduce the total volume of drug required to be injected for anesthetizing the plexus, thereby decreasing the chances of systemic toxicity of local anesthetics. The addition of dexamethasone to regional anesthesia with local anesthetics has added a newer aspect to medical uses of corticosteroids. Steroids have very strong anti-inflammatory and immunosuppressive effects. Perineural injection was found to be safe, devoid of adverse effects.

Dexamethasone is a preferred synthetic glucocorticoid as it is 25-30 times more potent than hydrocortisone and lacks any mineralocorticoid activity. In terms of sensory block onset. This was found to be clinically highly significant with $\mathrm{P}<0.0001$. The results of our study are similar to the results of a study conducted by Dar et al. [3] who added dexamethasone to ropivacaine and observed that the onset time in dexamethasone group for sensory block was $14.65+$ 3.31 min which was significantly shorter than the control group $(17.5+4.2 \mathrm{~min})$.

In a meta- analysis of RCTs by Huynh et al. [4] the authors studied 12 trials where dexamethasone was added to local anesthetic. They concluded that the time of onset of sensory block was significantly reduced from $10 \mathrm{~min}$ (control group) by weighted mean difference (Weighted Mean Difference - 78 s, 95\% confidence interval, $\mathrm{P}<0.001)$ with the addition of dexamethasone to local anesthetic. This was in concordance with the results of our study. In our study, the mean time for the onset of motor block was $17.53 \pm$ 1.70 in Group BS and 14.63 \pm 2.79 in Group BD.

In a study conducted by El- Baradey and Elshmaa,[1] they observed the onset time for motor block with bupivacaine and dexamethasone to be $11.4 \pm$ $3.6 \mathrm{~min}$. This result is similar to the findings of our study.

In our study, the mean duration of sensory block was $289.50 \pm 45.71 \mathrm{~min}$ in Group BS and $1160 \pm$ $143.10 \mathrm{~min}$ in Group BD. This difference was highly statistically significant with $\mathrm{P}<0.0001$. The mean duration of motor block was $216.27 \pm 37.73 \mathrm{~min}$ in Group BS and $870.87 \pm 101.14$ min in Group BD. This too was found to be highly statistically significant with $\mathrm{P}<0.0001$. This result was similar to a study by Choi et al. [5] who conducted a meta- analysis of RCTs and included nine trials including 801 patients. They observed that dexamethasone prolonged the analgesic duration for long- acting local anesthetic from 730 to 1306 min, while motor block was prolonged from 664 to 1102 min without any observed adverse events. The minor differences in the results between our studies can be due to the use of varying concentrations of dexamethasone, i.e., 4-10 mg, whereas we used $8 \mathrm{mg}$ for all patients in Group BD.

Similar results were also seen in a study by Vieira et al. [6] who in a randomized study evaluated the effect of dexamethasone on analgesia with $0.5 \%$ bupivacaine with epinephrine and clonidine [7]. The median sensory block duration was 1457 versus 833 $\min (\mathrm{P}<0.0001)$ and motor block duration was 1374 versus 827 min $(\mathrm{P}<0.0001)$ when compared with the control. These results though similar are slightly higher than ours. This difference could be due to the added vasoconstrictive effects of epinephrine and also due to the addition of clonidine. 
Albrecht et al. [8] in their study concluded that addition of dexamethasone to local anesthetic prolonged the duration of both sensory and motor block. The median duration of analgesia (sensory blockade) was increased by 488 mins, when dexamethasone was combined with long action local anesthetics. This result is in concordance with our findings where the difference due to adding dexamethasone was 871 mins for sensory block. The difference can be because their study included RCTs on all peripheral blocks and also that they studied and compiled the results considering the overall local anesthetics, i.e., both short and medium duration on one side and long duration on the other side, whereas we studied only bupivacaine which is a long- acting local anesthetic in only supraclavicular block. Another source of difference in the results can be that the inclusion of multiple studies caused the nerve location methods to vary, i.e., landmarks, nerve stimulator, or ultrasound, whereas in our study, we standardized it using USG for nerve location in both groups of patients. Biradar et al. [7] in their study concluded that dexamethasone caused prolongation of action of lignocaine for both sensory and motor blocks. This is similar to findings in our study where the action of bupivacaine was prolonged. The addition of dexamethasone induces vasoconstriction, blocks ectopic neuronal discharges, and prevents the release of inflammatory mediators. The prolonged duration of sensory and motor blockade and analgesia observed in dexamethasone group can be attributed to the fact that dexamethasone increases the activity of inhibitory potassium channels on nociceptive C- fibers (via glucocorticoid receptors), reducing their activity and prolonging local anesthetic activity. The different mechanisms of action of the two drugs when combined have an additive effect. The hemodynamic parameters such as heart rate, systolic BP, and diastolic BP were stable in both the groups in our study. This was similar to the results of the study conducted by El- Baradey and Elshmaa [1]. Thus, ultrasound- guided supraclavicular brachial plexus block using $0.5 \%$ bupivacaine and $8 \mathrm{mg}$ dexamethasone can provide adequate intra $^{-}$and postoperative analgesia when used for patients undergoing upper- limb surgeries.

\section{CONCLUSION}

Dexamethasone shortens the onset and prolongs the duration of sensory and motor blockade effectively and enhances the quality of blockade when used as an adjuvant to bupivacaine in supraclavicular block, with minimal hemodynamic changes, thus making dexamethasone a potential adjuvant for peripheral blocks.

\section{REFERENCES}

1. El-Baradey GF, Elshmaa NS. The efficacy of adding dexamethasone, midazolam, or epinephrine to $0.5 \%$ bupivacaine in supraclavicular brachial plexus block. Saudi journal of anaesthesia. 2014;8(Suppl 1):S78-83.

2. Lee JA, Atkinson RS, Rushman GB, NJ. D. Lee's Synopsis of Anaesthesia. . 11th ed. ed: Oxford: Buttersworth- Heinmann Ltd.1993.

3. Dar F, Najar M, Jan N. Effect of addition of dexamethasone to ropivacaine in supraclavicular brachial plexus block. Indian Journal of Pain. 2013;27(3):165-9.

4. Gupta A, Gupta A, Yadav N. Effect of dexamethasone as an adjuvant to ropivacaine on duration and quality of analgesia in ultrasoundguided transversus abdominis plane block in patients undergoing lower segment cesarean section - A prospective, randomised, single-blinded study. Indian J Anaesth. 2019;63(6):469-74.

5. Das A, Majumdar S, Halder S, Chattopadhyay S, Pal S, Kundu R, et al. Effect of dexmedetomidine as adjuvant in ropivacaine-induced supraclavicular brachial plexus block: A prospective, doubleblinded and randomized controlled study. Saudi journal of anaesthesia. 2014;8(Suppl 1):S72-7.

6. Vieira PA, Pulai I, Tsao GC, Manikantan P, Keller B, Connelly NR. Dexamethasone with bupivacaine increases duration of analgesia in ultrasoundguided interscalene brachial plexus blockade. European journal of anaesthesiology. 2010;27(3):285-8.

7. Duma A, Urbanek B, Sitzwohl C, Kreiger A, Zimpfer M, Kapral S. Clonidine as an adjuvant to local anaesthetic axillary brachial plexus block: a randomized, controlled study. British journal of anaesthesia. 2005;94(1):112-6.

8. Albrecht E, Kern C, Kirkham KR. A systematic review and meta-analysis of perineural dexamethasone for peripheral nerve blocks. Anaesthesia. 2015;70(1):71-83. 